\title{
Wide Variation and Rising Utilization of Stroke Magnetic Resonance Imaging: Data from 11 States
}

\author{
James F. Burke, MD, 1,2,3 Kevin A. Kerber, MD, ${ }^{3}$ Theodore J. Iwashyna, MD, PhD, 1,4 \\ and Lewis B. Morgenstern, MD ${ }^{3,5}$
}

\begin{abstract}
Objective: Neuroimaging is an essential component of the acute stroke evaluation. Magnetic resonance imaging (MRI) is more accurate than computed tomography (CT) for the diagnosis of stroke, but is more costly and timeconsuming. We sought to describe changes in MRI utilization from 1999 to 2008.

Methods: We performed a serial cross-sectional study with time trends of neuroimaging in patients with a primary International Classification of Diseases, 9th Edition, Clinical Modification discharge diagnosis of stroke admitted through the emergency department in the State Inpatient Databases from 10 states. MRI utilization was measured by Healthcare Cost and Utilization Project criteria. Data were included for states from 1999 to 2008 where MRI utilization could be identified.

Results: A total of 624,842 patients were hospitalized for stroke in the period of interest. MRI utilization increased in all states. Overall, MRI absolute utilization increased 38\%, and relative utilization increased $235 \%$ (28\% of strokes in 1999 to $66 \%$ in 2008). Over the same interval, CT utilization changed little (92\% in 1999 to $95 \%$ in 2008). MRI use varied widely by state. In 2008, MRI utilization ranged from a low of $55 \%$ of strokes in Oregon to a high of $79 \%$ in Arizona. Diagnostic imaging was the fastest growing component of total hospital costs (213\% increase from 1999 to 2007).

Interpretation: MRI utilization during stroke hospitalization increased substantially, with wide geographic variation. Rather than replacing CT, MRI is supplementing it. Consequently, neuroimaging has been the fastest growing component of hospitalization cost in stroke. Recent neuroimaging practices in stroke are not standardized and may represent an opportunity to improve the efficiency of stroke care.
\end{abstract}

ANN NEUROL 2012;71:179-185

$\mathrm{N}$ euroimaging is an essential component of acute stroke evaluation. Although magnetic resonance imaging (MRI) has several clear advantages compared to computed tomography (CT) in stroke diagnosis, its role in acute stroke management has yet to be completely elucidated. Compared to CT, MRI is a more accurate test for acute ischemia $^{1-5}$ and comparably accurate for the detection of intracerebral hemorrhage. ${ }^{6}$ Additional advantages of MRI include the improved ability to detect subtle hemorrhagic changes, ${ }^{7,8}$ to identify stroke location, ${ }^{9}$ and in some cases to clarify stroke mechanism. ${ }^{10-12}$

Despite these advantages, downsides to MRI do exist. These include longer scan times and higher costs per scan compared to CT. In addition, there are few data demonstrating that MRI findings lead to changes in patient management versus CT alone. ${ }^{13}$ Further, no data yet demonstrate improved outcomes in stroke patients undergoing MRI compared with stroke patients who do not undergo MRI.

In this study, we sought to describe trends in MRI use over the 10-year period of 1999 to 2008 as a means of determining its changing role in routine stroke care and to assess for opportunities to optimize its use. We hypothesized that MRI utilization would increase concordant with overall trends in diagnostic imaging utilization. ${ }^{14}$ We further hypothesized that geographic variation in MRI utilization would exist because evidence-based guidelines did not preferentially recommend CT or MRI 
for stroke evaluation. ${ }^{15-17}$ Finally, given prominent societal concerns about health care costs, we sought to characterize costs of MRI and diagnostic imaging during stroke hospitalization. Understanding these trends is essential to the development and implementation of standardized and efficient neuroimaging strategies in stroke.

\section{Patients and Methods}

\section{Dataset and Study Population}

We examined all patients with a discharge diagnosis of stroke in a select group of states from 1999 through 2008. States were chosen to incorporate geographic, temporal, and racial variability within the constraints of data availability, consistent participation in State Inpatient Databases (SID), data cost, and the ability to identify MRIs. Our specific study population consisted of discharge data from Washington (1999-2008), New York (1999-2008), Arizona (1999-2008), Florida (19992005), Nebraska (2004-2008), Massachusetts (2004-2008), North Carolina (2004-2008), Iowa (2008), Wisconsin (2008), New Jersey (2008), and Oregon (2008).

This study analyzed hospital discharges from SID developed as part of the Healthcare Cost and Utilization Project (HCUP), funded by the Agency for Healthcare Research and Quality. ${ }^{18}$ SID consists of abstracts for all acute care discharges within a state for a given year. Discharge abstracts consist of basic demographics, payer information, hospitalization characteristics, charges, primary diagnoses, and procedural utilization.

We included all discharges for subjects $>18$ years of age in whom the primary discharge diagnosis was ischemic stroke by International Classification of Diseases, 9th Edition, Clinical Modification (ICD-9-CM; 433.x1, 434.x1, 436) ${ }^{19}$ admitted through the emergency department. This approach has been previously validated and found to have a positive predictive value of $88 \%$ and sensitivity of $74 \% .^{20}$

The University of Michigan Institutional Review Board determined this study to be exempt from formal review as it relied on publicly available data.

\section{Categorizing Comorbidities}

To understand how patient-level variables may influence which patients undergo MRI, we characterized secondary diagnoses in our population of stroke patients. Vascular risk factor and potential stroke mimic diagnoses were characterized using HCUP single-level Clinical Classification System (CSS ${ }^{21}$ definitions: hypertension (CCS 98, 99), diabetes (CCS 49, 50), hyperlipidemia (CCS 53), epilepsy (CCS 83), migraine (CCS 84). A stroke modified Charlson comorbidity index was created for each patient using previously described methods. ${ }^{22,23}$

\section{Measuring Neuroimaging Exposure}

We measured MRI and CT utilization using the criteria for HCUP's Magnetic Resonance Technology Utilization and CT Scan flags, respectively. ${ }^{24}$ These flags define whether a patient underwent any CT or MRI imaging study during their hospital- ization and are primarily defined by Uniform Billing (UB-92) revenue codes. UB-92 revenue codes are standardized codes that are automatically generated by hospital billing systems and reflect charges from specific revenue centers. ${ }^{25}$ HCUP has internally validated revenue codes for the identification of specific utilization groups, including MRI and $\mathrm{CT}^{26}$ This approach was used instead of a less reliable identification strategy relying onICD-9-CM procedure code alone, because ICD-9-CM strategies are unreliable for procedures that do not change Diagnosis-Related Group (DRG) assignment, ${ }^{25}$ and stroke DRGs are not impacted by whether patients undergo neuroimaging.

\section{Statistical Analysis}

To summarize the characteristics of the population, demographic information, stroke risk factors, and neuroimaging utilization were reported with percentages or means and standard deviations (SDs). Comparisons between those receiving MRI and not receiving MRI were made using $t$ tests and chi-square tests for continuous and categorical variables, respectively. To describe trends in neuroimaging utilization, we divided the number of patients receiving a neuroimaging study by the total number of stroke cases identified, stratifying by state and year. Significance of trends was assessed using 1-sample $t$ tests of change in imaging utilization per year to evaluate the null hypothesis that no change in utilization occurred. To account for imbalance in potentially relevant variables, adjusted estimates of MRI utilization by state and over time were generated using multivariate logistic regression accounting for clustering at the hospital level. In both analyses, we adjusted for age, gender, vascular risk factors (hypertension, diabetes, hyperlipidemia, atrial fibrillation), Charlson comorbidity index, and insurance status (Medicare vs Medicaid vs private insurance vs other insurance). Results were reported as mean posterior probabilities, with covariates set at population means.

To estimate the availability of MRI and impact of MRI availability on utilization, hospital-level MRI utilization patterns were analyzed. To minimize the impact of miscoded data, a hospital was defined as having established MRI access if 3 or more stroke patients received MRIs at that facility in a given year. This definition will likely underestimate actual MRI access, as some hospitals may have the capacity to obtain MRIs and not opt to use that capacity. We then calculated the annual proportion of stroke patients presenting to hospitals with established MRI access.

Next, we sought to quantify geographic variation at the state and hospital referral region (HRR) levels by calculating aggregate MRI utilization rates at both levels by year. HRRs represent discrete geographic regions defined by regional referral patterns for tertiary medical care defined by the presence of at least 1 hospital that performs major cardiovascular and neurosurgical procedures. ${ }^{27-29}$ To measure annual utilization, we aggregated patient-level records at the HRR level, calculated utilization over HRR, ranked the HRRs by utilization rate, and then calculated utilization by HRR quintile.

Finally, we estimated inflation-adjusted costs for the total hospitalization, UB-92 cost categories, MRI, and CT. Total 
TABLE 1: Study Population Baseline Characteristics

\begin{tabular}{llll}
\hline Demographics & MRI, $\mathbf{n}=\mathbf{3 1 0 , 7 6 8}$ & No MRI, $\mathbf{n}=\mathbf{3 1 4 , 0 7 4}$ & All Subjects, $\mathbf{n}=\mathbf{6 2 4 , 8 4 2}$ \\
\hline $\begin{array}{l}\text { Age, mean yr (SD) } \\
\text { Female }\end{array}$ & $70(14)$ & $76(13)$ & $73(14)$ \\
$\begin{array}{l}\text { Race/ethnicity } \\
\text { White }\end{array}$ & $57 \%$ & $54 \%$ \\
\hline Black & $60 \%$ & $61 \%$ & $61 \%$ \\
\hline Hispanic & $7 \%$ & $7 \%$ & $7 \%$ \\
\hline Insurance & $14 \%$ & $12 \%$ & $13 \%$ \\
\hline Medicare & & & $70 \%$ \\
\hline Medicaid & $64 \%$ & $75 \%$ & $7 \%$ \\
\hline Private insurance & $8 \%$ & $6 \%$ & $18 \%$ \\
\hline Other insurance & $22 \%$ & $14 \%$ & $5 \%$ \\
\hline Comorbidities & $6 \%$ & $4 \%$ & $69 \%$ \\
\hline Hypertension & & $67 \%$ & $25 \%$ \\
\hline Hyperlipidemia & $72 \%$ & $19 \%$ & $29 \%$ \\
\hline Diabetes & $32 \%$ & $28 \%$ & $24 \%$ \\
\hline Coronary artery disease & $30 \%$ & $27 \%$ & $21 \%$ \\
\hline Atrial fibrillation & $21 \%$ & $4.5(1.9)$ & $4.1(1.9)$ \\
\hline Modified Charlson, mean (SD) & $3.8(2)$ & & \\
\hline MRI = magnetic resonance imaging; SD & standard deviation. & & \\
\hline
\end{tabular}

hospital charges were abstracted directly from the SID discharge record. All charges within a given UB-92 cost center, identified by revenue code, were summed to derive charges per cost center. MRI and CT costs were calculated by summing costs associated with UB-92 revenue codes 610-619 and 0350-0359, respectively. This approach combines all CT- and MRI-related costs into single categories and consequently is not able to distinguish between the costs of repeat imaging studies and of combined imaging studies such as multimodal imaging. To account for local variation in charges, HCUP cost-to-charge ratios were applied to hospital charges to estimate hospital costs. ${ }^{30}$ As a consequence, we were able to estimate actual costs of care as opposed to the amount charged for care. Because we were interested in overall expenditure trends, costs were inflation-adjusted to 2008 dollars using the gross domestic product price index. ${ }^{31}$ For clarity of reporting, state data were aggregated into 2 separate time cohorts based on the availability of longitudinal data. Cohort 1 consists of data from New York, Florida, Arizona, and Washington from 1999 to 2007, and cohort 2 consists of data from New York, Arizona, Washington, North Carolina, and Massachusetts from 2004 to 2008. We report costs for the 5 largest cost centers. All costs were summarized as means and standard deviations. All data analyses were performed with Stata version 11.1 (Stata Corp, College Station, TX).

\section{Results}

\section{Study Population}

Over the 10-year study period (1999-2008), 624,842 primary ischemic stroke hospitalizations were identified (Table 1). Mean age was $73 \pm 14$ years, and 54\% were female. Cardiovascular risk factors were common. Most patients $(70 \%)$ were insured by Medicare.

MRI was performed in $50 \%$ of the total population and CT in 95\%. On average, patients who underwent MRI were younger (70 years vs 76 years, $p<0.001$ ), more likely to have private insurance $(22 \%$ vs $15 \%, p<$ 0.001 ), and had a greater burden of vascular risk factors compared with patients who did not undergo MRI. A history of atrial fibrillation was less common in patients who had an MRI ( $27 \%$ vs. $16 \%, p<0.001)$.

\section{Temporal Trends in Diagnostic imaging}

From 1999 to 2008, absolute MRI utilization increased $38 \%$, a relative increase of $235 \%$ (28\% in 1999 to $66 \%$ in 2008, $p=0.003$ for trend, Fig 1). Of patients receiving an MRI, 95\% also received a CT. Overall CT utilization did not change during the same interval $(92 \%$ in 1999 to $95 \%$ in 2008, $p=0.34$ for trend). After 

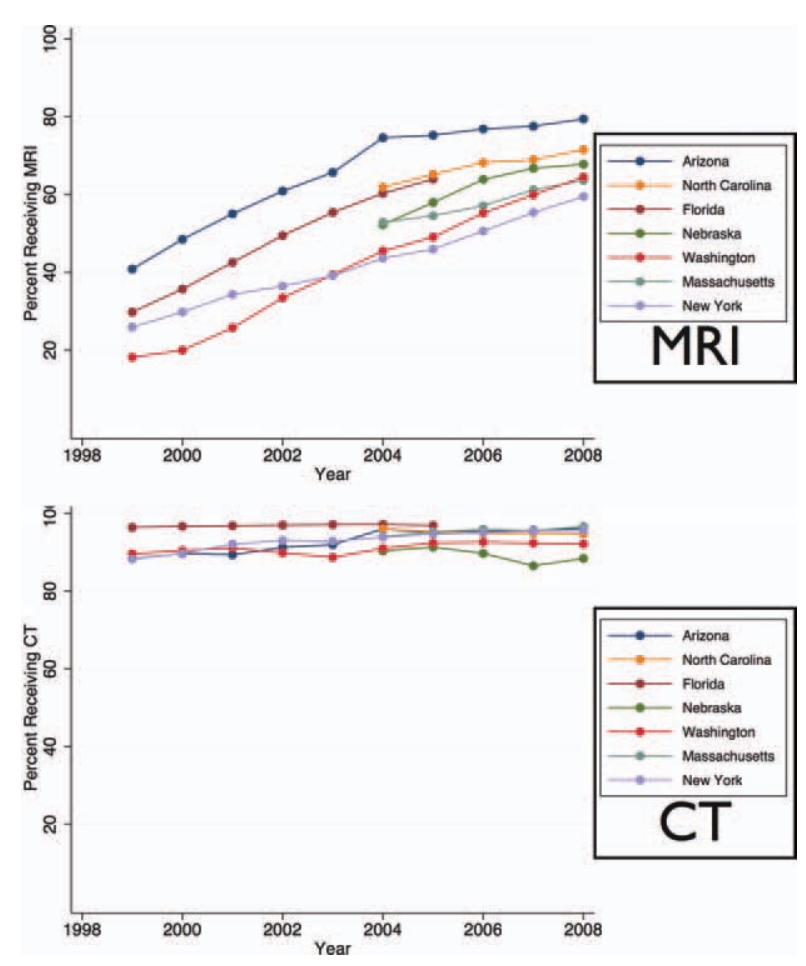

FIGURE 1: Utilization trends in diagnostic imaging for stroke. $\mathrm{CT}=$ computed tomography; MRI = magnetic resonance imaging.

adjusting for the changing age, sex, vascular risk factors, comorbidities, and insurance status of the population, each year was associated with a $4.0 \%$ increase the probability of receiving MRI in the logistic regression model (odds ratio, 1.20; 95\% CI, 1.18-1.22).

\section{Regional Variation in Diagnostic Imaging}

Utilization of MRI by state is illustrated in Figure 2 for 2008, the year for which the broadest cross section of

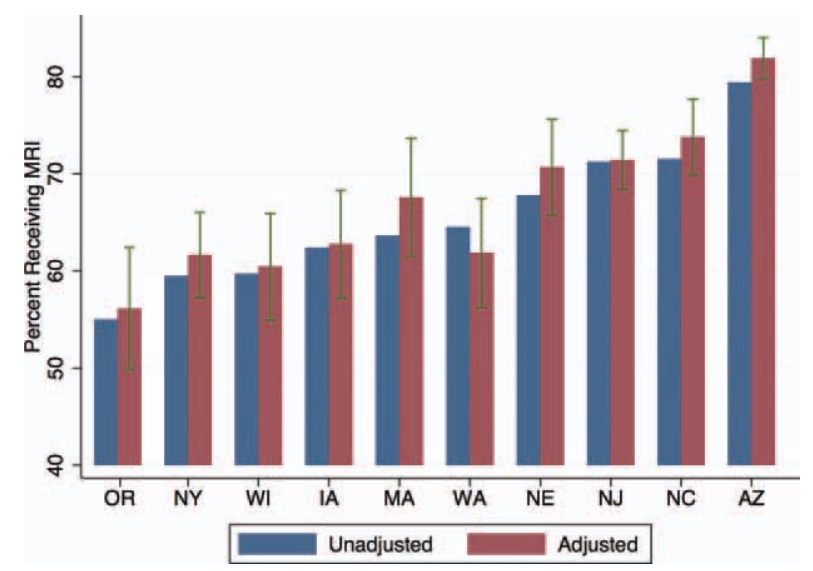

FIGURE 2: Percentage of stroke patients receiving magnetic resonance imaging (MRI), 2008. Blue bars represent unadjusted utilization by state. Red bars represent utilization adjusted for age, sex, vascular risk factors, comorbidities, and insurance status. data was available. In 2008, MRI utilization ranged from a low of $55 \%$ in Oregon to a high of $79 \%$ in Arizona. Adjustment for age, sex, vascular risk factors, comorbidities, and insurance status had minimal impact on the pattern of variation by state. More extreme geographic variation is seen when analyzing MRI utilization by HRR quintile (Table 2). In every year, MRI utilization rates were at least 30\% higher in the highest utilizing quintile compared to the lowest utilizing quintile.

\section{Established Access to MRI}

There was a modest overall increase in the number of hospitals with established MRI access; $74 \%$ of hospitals had access in 1999 versus 87\% in $2008(p<0.01$; Table $3)$. The hospitals that gained MRI access over time were mostly lower volume centers, consequently there was less change in the percentage of stroke patients presenting to hospitals with MRI access: $89 \%$ in 1999 versus $96 \%$ in $2008(p<0.01)$. This suggests that physician practice regarding MRI is more important than the availability of MRI scanners per se to explain the rising rates of MRI utilization.

\section{Cost Trends}

Mean hospital costs increased from \$9,058 (SD, $\$ 11,867$ ) to $\$ 12,842$ (SD, $\$ 15,551$ ) in the group of states with the most complete cost data (New York, Florida, Arizona, and Washington from 1997 to 2007). Diagnostic imaging (radiotherapy, CT, MRI, nuclear

\begin{tabular}{|c|c|c|c|c|c|}
\hline \multirow[t]{2}{*}{ Year } & \multicolumn{5}{|c|}{$\begin{array}{l}\text { Percentage Receiving MRI by } \\
\text { HRR Utilization Quintile }\end{array}$} \\
\hline & $\begin{array}{l}1 \text { (lowest } \\
\text { users) }\end{array}$ & 2 & 3 & 4 & $\begin{array}{l}5 \text { (highest } \\
\text { users) }\end{array}$ \\
\hline 1999 & $7 \%$ & $17 \%$ & $23 \%$ & $29 \%$ & $39 \%$ \\
\hline 2000 & $10 \%$ & $21 \%$ & $27 \%$ & $35 \%$ & $50 \%$ \\
\hline 2001 & $17 \%$ & $28 \%$ & $35 \%$ & $42 \%$ & $55 \%$ \\
\hline 2002 & $22 \%$ & $31 \%$ & $41 \%$ & $51 \%$ & $63 \%$ \\
\hline 2003 & $23 \%$ & $36 \%$ & $45 \%$ & $57 \%$ & $66 \%$ \\
\hline 2004 & $31 \%$ & $45 \%$ & $51 \%$ & $64 \%$ & $72 \%$ \\
\hline 2005 & $34 \%$ & $47 \%$ & $55 \%$ & $66 \%$ & $74 \%$ \\
\hline 2006 & $34 \%$ & $52 \%$ & $55 \%$ & $62 \%$ & $77 \%$ \\
\hline 2007 & $36 \%$ & $55 \%$ & $60 \%$ & $66 \%$ & $77 \%$ \\
\hline 2008 & $43 \%$ & $58 \%$ & $64 \%$ & $70 \%$ & $78 \%$ \\
\hline
\end{tabular}




\begin{tabular}{|c|c|c|}
\hline Year & $\begin{array}{l}\text { Percentage of } \\
\text { Hospitals with } \\
\text { MRI Access }\end{array}$ & $\begin{array}{l}\text { Percentage of } \\
\text { Stroke Patients } \\
\text { at Hospital with } \\
\text { MRI Access }\end{array}$ \\
\hline 1999 & $74 \%$ & $89 \%$ \\
\hline 2000 & $77 \%$ & $91 \%$ \\
\hline 2001 & $80 \%$ & $93 \%$ \\
\hline 2002 & $83 \%$ & $94 \%$ \\
\hline 2003 & $82 \%$ & $94 \%$ \\
\hline 2004 & $83 \%$ & $94 \%$ \\
\hline 2005 & $86 \%$ & $95 \%$ \\
\hline 2006 & $83 \%$ & $94 \%$ \\
\hline 2007 & $84 \%$ & $94 \%$ \\
\hline 2008 & $87 \%$ & $96 \%$ \\
\hline
\end{tabular}

medicine, ultrasound, electrocardiography, and electroencephalography) was the second largest cost center over this interval behind only room and board charges, and underwent the largest percentage increase (213\%; Fig 3). MRI costs increased faster than overall diagnostic imaging costs, increasing 413\% from 1999 to 2007, from 3\% of total hospital costs in 1999 to $9 \%$ in 2007. CT costs increased by $171 \%$ from $6 \%$ of total costs in 1999 to $8 \%$ in 2007. Similar trends were seen in the group of states with adequate cost data only in the later period (New York, Arizona, Washington, North Carolina, and Massachusetts from 2004 to 2008); diagnostic imaging was the second largest cost component and underwent the largest percentage increase (129\%), with MRI (138\%) and CT costs (133\%) increasing slightly faster. In 2008, MRI costs accounted for $10 \%$ of total hospital costs and CT for $8 \%$.

\section{Discussion}

MRI utilization in ischemic stroke has increased substantially from 1999 to 2008. Growth in MRI utilization was seen in every year in every state in our study population. In high-utilizing regions, the rate has plateaued at a level suggesting that nearly all eligible patients are receiving MRI. ${ }^{32,33}$ However, $95 \%$ of patients receiving MRI also received CT. MRI is not replacing CT as the primary stroke neuroimaging study; instead, receipt of multiple neuroimaging studies is increasingly common. In addition to an increase in overall MRI utilization, we also observed marked geographic variation. In 2008,
$55 \%$ of stroke patients underwent MRI in Oregon compared to $79 \%$ in Arizona. Partly as a consequence of this widespread increased use, diagnostic imaging has been the fastest growing cost component of stroke care. These data suggest that neuroimaging practices in stroke are neither standardized nor efficient.

The wide geographic variation in MRI utilization demonstrates a potential opportunity to improve stroke care by increasing standardization. This variation was not accounted for by measurable patient characteristics or hospital acquisition of MRI technology. Although we cannot directly measure patient preferences, there is little evidence that patients have strong and variable preferences among imaging modalities, independent of the advice of their physicians ${ }^{34}$; it is further unclear why those preferences would be so geographically variable as to explain the observed pattern. Absent a credible alternative explanation, variation in physician practice patterns likely accounts for much of the variation in MRI utilization.

The existence of variation in physician practice in this context is not surprising, as variation tends be greater when the evidence for a practice is less certain, ${ }^{29}$ and stroke imaging guidelines during the study period did not preferentially recommend CT or MRI. ${ }^{15,17}$ Recent guidelines have generally made more favorable recommendations regarding MRI than previous guidelines, but
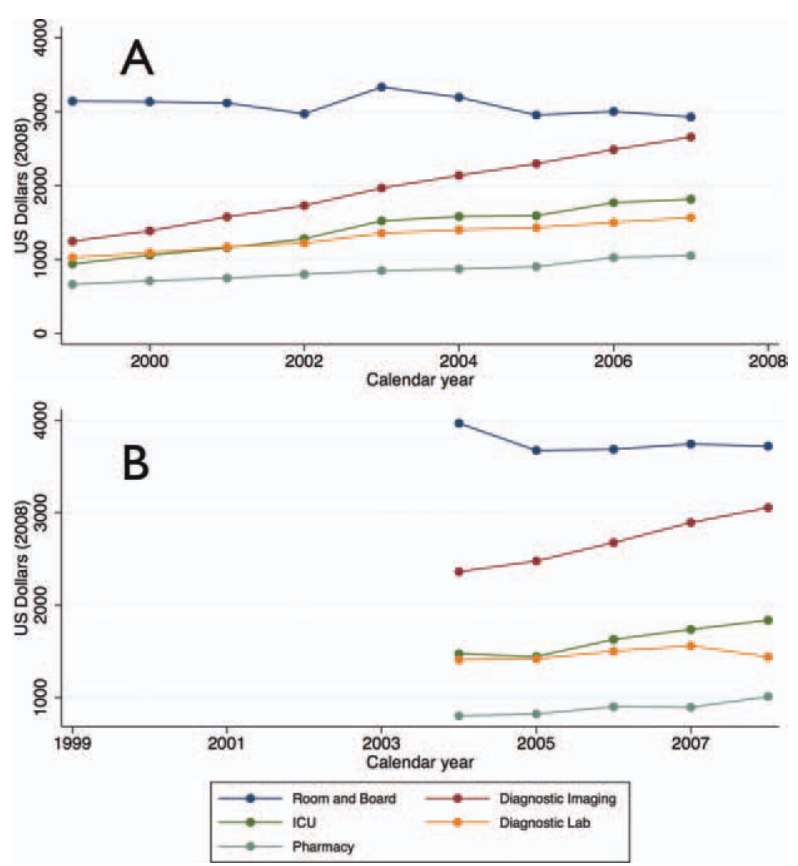

FIGURE 3: Trends in stroke cost components. (A) Trends in cost components from 1999 to 2007 in New York, Florida, Arizona, and Washington. (B) Trends in cost components from 2004 to 2008 in New York, Arizona, Washington, North Carolina, and Massachusetts. ICU = intensive care unit. 
inconsistency between these guidelines exists. Recently, an American Heart Association scientific statement was the first major guideline to recommend routine use of MRI or CT angiography for stroke patients. ${ }^{35}$ The recent American Academy of Neurology guideline on the role of MRI in stroke ${ }^{36}$ limits its preferential recommendation of MRI to the period within 12 hours of stroke onset. The most recent European Stroke Organization guideline $^{37}$ calls for stroke patients to receive either CT or MRI. Standardization of neuroimaging practices may improve as guidelines become more consistent, particularly if evidence emerges that MRI leads to improved outcomes or more optimal physician decision making. The current highly variable use of MRI suggests that there may be community equipoise that would make a randomized trial ethical, feasible, and useful.

Not only are stroke neuroimaging practices not standardized, but our findings suggest stroke neuroimaging may be unnecessarily costly. In our sample, 95\% of patients receiving a MRI also received a CT; thus, minimizing the use of multiple imaging studies may represent a viable strategy to contain neuroimaging costs. If the practice of obtaining both MRI and CT reflects physician preference for MRI in a context where CT can be obtained more quickly, then several broad strategies for increasing efficiency are possible. First, some patients may be able to safely wait for an imaging study, allowing MRI to be performed without a preceding CT. Patients with delayed presentations who are out of the acute treatment window and have stable examinations might meet these criteria and represent a significant proportion of all stroke patients. Up to $36 \%$ of stroke patients present $>12$ hours after onset. ${ }^{38}$ Second, wider dissemination of rapid stroke MRI protocols relying on a selective set of sequences (ie, diffusion-weighted, gradient-echo, and T2-weighted sequences) may minimize resource utilization and allow for quicker scanner turnover, thus enabling earlier MRI acquisition. ${ }^{39}$ For MRI to be a viable alternative to $\mathrm{CT}$ in the acute stroke period, scan times for MRI must not be greater than CT to minimize time delay for thrombolysis.

This study has several important limitations. First, the use of administrative data to identify stroke is an imprecise process, raising the possibility that some of the variability in MRI utilization and cost may be due to imprecision in coding of stroke diagnoses. To minimize this, we have used a previously validated algorithm to identify stroke cases. ${ }^{19}$ Similarly, revenue code-based identification of imaging studies is also limited by the inability to reliably identify the subcomponents of an imaging study, the number of times an individual patient received a given neuroimaging study, which body part was imaged, and the timing of imaging studies. Consequently, we are unable to reliably determine the role of magnetic resonance angiography compared to MRI, which portion of studies were focused on the central nervous system, or when imaging studies were obtained during the hospitalization. Because of this limited information, we cannot speculate as to why physicians obtained an MRI in an individual case (ie, was MRI obtained to aid thrombolysis decision making, clarify diagnosis, or inform secondary prevention strategies?). Additionally, although our dataset was selected to maximize geographic and demographic variability, the Southern states and African Americans are underrepresented.

The use of MRI in ischemic stroke has substantially increased over the past decade, with wide geographic variation and increasing contribution to the cost of stroke care. These findings emphasize the importance of future research to define which stroke patients are likely to benefit from MRI, how MRI information should be applied to individuals, and the relationship between MRI and clinically meaningful outcomes. Finally, these findings illustrate the potential to reduce variability and improve the efficiency of neuroimaging in stroke.

\section{Acknowledgments}

This work was supported by the Robert Wood Johnson Foundation Clinical Scholars Program and an associated Veterans Affairs Advanced Fellowship to J.F.B. It was also supported by the NIH via K08 HL091249 to T.J.I. K.A.K. received research support from NIH grant K23 RR024009 and Agency for Healthcare Research and Quality (AHRQ) grant R18 HS017690. L.B.M. received research support from NIH grant R01NS038916 and AHRQ grant R18 HS017690.

We thank Dr C. Cooke for his assistance in assembling and using the dataset.

\section{Authorship}

J.F.B. had full access to all of the data in the study, performed the primary analyses, and takes responsibility for the integrity of the data and the accuracy of the data analysis.

\section{Potential Conflicts of Interest}

Nothing to report.

\section{References}

1. Brazzelli M, Sandercock PA, Chappell FM, et al. Magnetic resonance imaging versus computed tomography for detection of acute vascular lesions in patients presenting with stroke symptoms. Cochrane Database Syst Rev 2009;(4):CD007424. 
2. Barber PA, Darby DG, Desmond PM, et al. Identification of major ischemic change. Diffusion-weighted imaging versus computed tomography. Stroke 1999;30:2059-2065.

3. Fiebach JB, Schellinger PD, Jansen $\mathrm{O}$, et al. $\mathrm{CT}$ and diffusionweighted MR imaging in randomized order: diffusion-weighted imaging results in higher accuracy and lower interrater variability in the diagnosis of hyperacute ischemic stroke. Stroke 2002;33:2206-2210.

4. Chalela J, Kidwell C, Nentwich L, Luby M. Magnetic resonance imaging and computed tomography in emergency assessment of patients with suspected acute stroke: a prospective comparison. Lancet 2007;369:293-298.

5. González RG, Schaefer PW, Buonanno FS, et al. Diffusion-weighted MR imaging: diagnostic accuracy in patients imaged within 6 hours of stroke symptom onset. Radiology 1999;210:155-162.

6. Morgenstern LB, Hemphill JC, Anderson C, et al. Guidelines for the management of spontaneous intracerebral hemorrhage: a guideline for healthcare professionals from the American Heart Association/ American Stroke Association. Stroke 2010;41:2108-2129.

7. Wardlaw JM, Keir SL, Seymour J, et al. What is the best imaging strategy for acute stroke? Health Technol Assess 2004;8: iii,ix-x,1-180.

8. Bryan R, Levy L, Whitlow W, et al. Diagnosis of acute cerebral infarction: comparison of CT and MR imaging. Am J Neuroradiol $1991 ; 12: 611$.

9. Schulz UG, Briley D, Meagher T, et al. Diffusion-weighted MRI in 300 patients presenting late with subacute transient ischemic attack or minor stroke. Stroke 2004;35:2459-2465.

10. Barber PA. Is diffusion-weighted imaging helpful in determining the source of stroke? Commentary. Nat Rev Neurol 2006;2:424-425.

11. Gass A, Ay H, Szabo K, Koroshetz WJ. Diffusion-weighted MRI for the "small stuff": the details of acute cerebral ischaemia. Lancet Neurol 2004;3:39-45.

12. Wessels T, Wessels C, Ellsiepen A, et al. Contribution of diffusionweighted imaging in determination of stroke etiology. AJNR Am J Neuroradiol 2006:27:35-39.

13. Albers GW, Lansberg MG, Norbash AM, et al. Yield of diffusionweighted MRI for detection of potentially relevant findings in stroke patients. Neurology 2000;54:1562-1567.

14. Medicare: trends in fees, utilization, and expenditures for imaging services before and after implementation of the Deficit Reduction Act of 2005. Washington, DC: Government Accountability Office, 2008.

15. Adams HP Jr, Del Zoppo GJ, Alberts MJ, et al. Guidelines for the early management of adults with ischemic stroke: a guideline from the American Heart Association/American Stroke Association Stroke Council, Clinical Cardiology Council, Cardiovascular Radiology and Intervention Council, and the Atherosclerotic Peripheral Vascular Disease and Quality of Care Outcomes in Research Interdisciplinary Working Groups: the American Academy of Neurology affirms the value of this guideline as an educational tool for neurologists. Stroke 2007;38:1655-1711.

16. Culebras A, Kase CS, Masdeu JC, et al. Practice guidelines for the use of imaging in transient ischemic attacks and acute stroke. A report of the Stroke Council, American Heart Association. Stroke 1997;28:1480-1497.

17. Olsen TS, Langhorne P, Diener HC, et al. European Stroke Initiative recommendations for stroke management-update 2003. Cerebrovasc Dis 2003;16:311-337.

18. HCUP state inpatient databases. Rockville, MD: Agency for Healthcare Research and Quality, 1999-2009. Available at: http:// www.hcup-us.ahrq.gov/sidoverview.jsp. Accessed August 31, 2011.

19. Goldstein LB. Accuracy of ICD-9-CM coding for the identification of patients with acute ischemic stroke: effect of modifier codes. Stroke 1998:29:1602-1604.

20. Tirschwell DL, Longstreth WT. Validating administrative data in stroke research. Stroke 2002;33:2465-2470.
21. HCUP clinical classifications software for ICD-9-CM. Rockville, MD: Agency for Healthcare Research and Quality. Available at: http:// www.hcup-us.ahrq.gov/toolssoftware/ccs/ccs.jsp. Accessed August 31, 2011.

22. Quan H, Sundararajan V, Halfon $P$, et al. Coding algorithms for defining comorbidities in ICD-9-CM and ICD-10 administrative data. Med Care 2005;43:1130-1139.

23. Goldstein LB, Samsa GP, Matchar DB, Horner RD. Charlson index comorbidity adjustment for ischemic stroke outcome studies. Stroke 2004;35:1941-1945

24. HCUP utilization flags. Rockville, MD: Agency for Healthcare Research and Quality, 2003. Available at: http://www.hcup-us. ahrq gov/toolssoftware/util_flags/utilflag.jsp. Accessed August 31, 2011.

25. Dismuke CE. Underreporting of computed tomography and magnetic resonance imaging procedures in inpatient claims data. Med Care 2005;43:713-717

26. Elixhauser A, Barrett M, Nisbet J. Development of utilization flags for use with UB-92 administrative data. Rockville, MD: Agency for Healthcare Research and Quality, 2006. Available at: http://www.hcup-us. ahrq.gov/reports/methods.jsp. Accessed date February 16, 2011.

27. Wennberg JE, Cooper MM, eds. The Dartmouth atlas of health care 1999. Chicago, IL: American Hospital Publishing, 1999.

28. Appendix on the geography of health care in the United States. Available at: http://www.dartmouthatlas.org/downloads/methods/ geogappdx.pdf. Accessed on February 16, 2011

29. Sirovich B, Gallagher PM, Wennberg DE, Fisher ES. Discretionary decision making by primary care physicians and the cost of U.S. health care. Health Affairs 2008;27:813-823.

30. HCUP cost-to-charge ratio files (CCR). Rockville, MD: Agency for Healthcare Research and Quality, 2001-2008. Available at: http:// www.hcup-us.ahrq.gov/db/state/costtocharge.jsp. Accessed August 31,2011

31. Using appropriate price indices for analyses of health care expen ditures or income across multiple years. Rockville, MD: Medical Expenditure Panel Survey. Available at: http://www.meps.ahrq. gov/mepsweb/about_meps/Price_Index.shtml. Accessed date February 16, 2011.

32. Singer OC, Sitzer M, du Mesnil de Rochemont R, NeumannHaefelin T. Practical limitations of acute stroke MRI due to patient-related problems. Neurology 2004;62:1848-1849.

33. Hand PJ, Wardlaw JM, Rowat AM, et al. Magnetic resonance brain imaging in patients with acute stroke: feasibility and patient related difficulties. J Neurol Neurosurg Psychiatry 2005;76: 1525-1527.

34. Anthony DL, Herndon MB, Gallagher PM, et al. How much do patients' preferences contribute to resource use? Health Aff (Millwood) 2009;28:864-873

35. Latchaw RE, Alberts MJ, Lev $\mathrm{MH}$, et al. Recommendations for imaging of acute ischemic stroke. a scientific statement from the American Heart Association. Stroke 2009;40:3646-3678.

36. Schellinger PD, Bryan RN, Caplan LR, et al. Evidence-based guideline: the role of diffusion and perfusion MRI for the diagnosis of acute ischemic stroke: report of the Therapeutics and Technology Assessment Subcommittee of the American Academy of Neurology. Neurology 2010;75:177-185.

37. European Stroke Organisation (ESO) Executive Committee; ESO Writing Committee. Guidelines for management of ischaemic stroke and transient ischaemic attack 2008. Cerebrovasc Dis 2008; 25:457-507.

38. Majersik JJ, Smith MA, Zahuranec DB, et al. Population-based analysis of the impact of expanding the time window for acute stroke treatment. Stroke 2007;38:3213-3217.

39. Schellinger PD, Jansen $O$, Fiebach JB, et al. Feasibility and practicality of MR imaging of stroke in the management of hyperacute cerebral ischemia. Am J Neuroradiol 2000;21:1184. 